\title{
Observations of high and low Fe charge states in individual solar wind streams with coronal-hole origin
}

\author{
Verena Heidrich-Meisner, Thies Peleikis, Martin Kruse, Lars Berger, and Robert Wimmer-Schweingruber
}

Christian Albrechts University at Kiel, 24118 Kiel, Germany

e-mail: heidrich@physik.uni-kiel.de

Received 18 December 2015 / Accepted 18 July 2016

\begin{abstract}
Context. The solar wind originating from coronal holes is comparatively well-understood and is characterized by lower densities and average charge states compared to the so-called slow solar wind. Except for wave perturbations, the average properties of the coronal-hole solar wind are passably constant.

Aims. In this case study, we focus on observations of the Solar Wind Ion Composition Spectrometer (SWICS) on the Advanced Composition Explorer (ACE) of individual streams of coronal-hole solar wind that illustrate that although the $\mathrm{O}$ and $\mathrm{C}$ charge states are low in coronal-hole wind, the Fe charge distribution is more variable. In particular, we illustrate that the Fe charge states in coronal-hole solar wind are frequently as high as in slow solar wind.

Methods. We selected individual coronal-hole solar wind streams based on their collisional age as well as their respective $\mathrm{O}$ and $\mathrm{C}$ charge states and analyzed their Fe charge-state distributions. Additionally, with a combination of simple ballistic back-mapping and the potential field source surface model, transitions between streams with high and low Fe charge states were mapped back to the photosphere. The relative frequency of high and low Fe charge-state streams is compared for the years 2004 and 2006.

Results. We found several otherwise typical coronal-hole streams that include Fe charge states either as high as or lower than in slow solar wind. Eight such transitions in 2006 were mapped back to equatorial coronal holes that were either isolated or connected to the northern coronal-hole. Attempts to identify coronal structures associated with the transitions were so far inconclusive.
\end{abstract}

Key words. solar wind - Sun: heliosphere - Sun: magnetic fields

\section{Introduction}

The steady solar wind is typically divided into two dominant types, fast and slow solar wind. However, the differences in their properties are better ordered by elemental and charge-state abundances rather than by solar wind speed. Here, we focus on fast solar wind. It has been uniquely identified as originating from coronal holes and the release mechanism is well understood (i.e. Tu et al. 2005). Therefore, in the following, we use the term coronal-hole wind instead of fast solar wind. Aside from fluctuations caused by waves (mainly Alfvénic waves), its plasma and compositional properties are constant (e.g. von Steiger et al. 2000). Both the elemental and charge-state compositions of the solar wind reflect the conditions in the respective solar source regions. In particular, the charge-state distribution for each solar wind ion species is (almost completely) determined in the corona. For each ion pair the recombination and ionization rates are temperature dependent and the hot corona allows high ionization states to occur. For a pair of adjacent ionization states $i \leftrightarrow i+1$, this can be expressed by a temperature dependent charge modification time scale $\tau_{\text {mod, } i}(T)=\frac{1}{n_{\mathrm{e}}\left(C_{i}+R_{i+1}\right)}$, where $T$ denotes the electron temperature in $\mathrm{K}, n_{\mathrm{e}}$ the electron density, $C_{i}$ the ionization rate of the $i$ th ionization state, and $R_{i+1}$ the recombination rate of the $(i+1)$ th to the $i$ th ionization state. But the ionization state is not only temperature dependent. A sufficiently high electron density is required to allow recombination. Thus, a simple model to explain the observed solar wind speeds and charge states assumes that the charge state can change along the solar-wind flux tube until the expansion time scale (which depends on the electron density profile in the corona) is of the same order as the charge modification time scale of an ion pair. Beyond this point the charge-state distribution remains "frozenin" as the solar wind propagates further outwards.

The coronal-hole wind is known for comparatively low $\mathrm{O}$ and $\mathrm{C}$ charge states and corresponding freeze-in temperatures. They are particularly low compared to those of the slow solar wind. The $\mathrm{O}$ charge-state distribution can be considered as a tracer for the solar wind type. The ratio $n_{\mathrm{O}^{7+}} / n_{\mathrm{O}^{6+}}$ of the densities of $\mathrm{O}^{7+}$ to $\mathrm{O}^{6+}$ (denoted with $n_{\mathrm{O}^{7+}}$ and $n_{\mathrm{O}^{6+}}$, respectively) has been frequently used in solar wind categorization schemes (e.g. Zurbuchen et al. 2002; Zhao et al. 2009) to differentiate between fast (coronal hole) and slow solar wind. In accordance with the cool $\mathrm{O}$ and $\mathrm{C}$ signatures in coronal-hole wind, the charge-state distributions of other ions, for example Fe, could also be expected to be cooler in the coronal-hole wind than in the slow solar wind. However, that is clearly not necessarily the case. While the Ulysses observations in von Steiger et al. (2000), Richardson (2014) and Zhao \& Landi (2014, as well as the ACE observations in Zhao \& Landi 2014) show on average higher Fe charge states in the fast solar wind than in the slow solar wind (see for example Plate 5 in von Steiger et al. 2000), the STEREO results (Galvin et al. 2009) indicate lower Fe charge states in coronal-hole wind. For example, for the solarwind speed bin $650-700 \mathrm{~km} \mathrm{~s}^{-1}$ the average Fe charge state in coronal-hole wind observed with PLASTIC on STEREO A from 2007-2009 is given as 9.25, which is lower than for all slow 
solar wind bins considered in that article. For all other high speed bins, the average Fe charge state is even lower. Zhao \& Landi (2014) compared long-term properties of coronal-hole wind at different solar minima and identified two subcategories, coronalhole wind originating from polar coronal holes and coronal-hole wind originating from the equatorial region. Lower Fe charge states were observed in equatorial coronal-hole wind than in polar coronal holes and additionally lower charge states were found in the second solar minimum. A gradual charge-state decrease for $\mathrm{O}, \mathrm{C}$, and Fe from solar maximum to the following solar minimum in solar cycle 23 has been discussed in Lepri et al. (2013) and Zhao \& Fisk (2010) underlines differences in the composition of the slow solar wind between the two consecutive solar minima in solar cycles 22 and 23.

Instead of focusing on the statistics of charge-state parameters gathered over long time periods, we discuss several case studies of individual streams within the ACE/SWICS data that show regions of high and low Fe charge states with a clear transition between these regions. We then map these streams back to their coronal sources and assess whether there are any coronal structures that may be associated with these transitions.

\section{Data analysis and event selection}

The SWICS instrument on ACE (Gloeckler et al. 1998) combines a time-of-flight mass spectrometer and energy-per-charge analyzer with an energy measurement. A detailed and extensive description of the data analysis procedure applied to the pulse height amplitude (PHA) data is given in the PhD thesis Berger (2008) and has been applied in, e.g. Berger et al. (2011).

The proton density $n_{\mathrm{p}}$ (from the Solar Wind Electron, Proton and Alpha Monitor (SWEPAM) on ACE, McComas et al. 1998), and the densities of $\mathrm{O}^{6+}, \mathrm{O}^{7+}, \mathrm{C}^{5+}$, and $\mathrm{C}^{6+}$ are used for the characterization of solar-wind plasma as coronal-hole wind. We selected coronal-hole-wind streams based on four-hour resolution data from SWICS and SWEPAM and the following three criteria: (1) Low $\mathrm{O}$ and $\mathrm{C}$ charge-state ratios $\left(n_{\mathrm{O}^{7+}} / n_{\mathrm{O}^{6+}}\right.$ and $n_{\mathrm{C}^{6+}} / n_{\mathrm{C}^{5+}}$, respectively) are considered as the decisive property for identifying coronal-hole wind. Zhao et al. (2009) proposed an upper limit of $n_{\mathrm{O}^{7+}} / n_{\mathrm{O}^{6+}}<0.145$ for coronal-hole wind. To avoid potential contamination with inter-stream solar wind, we apply $n_{\mathrm{O}^{7+}} / n_{\mathrm{O}^{6+}}<0.1$ which was also used in Zurbuchen et al. (2002). For C, we adopted an upper threshold of $n_{\mathrm{C}^{6+}} / n_{\mathrm{C}^{5+}}<1$. (2) Based on the observations in Kasper et al. (2008), we additionally require a low collisional age $a_{\mathrm{col}}=\frac{r}{v_{\mathrm{p}} \tau_{\mathrm{col}}}<0.1$, with $r$ as the distance from the Sun to ACE, $v_{\mathrm{p}}$ the solar-wind proton speed, and $\tau_{\text {col }}$ the time scale for $\alpha$ to proton energy exchange due to small-angle Coulomb scattering, where $\tau_{\text {col }} \sim n_{\mathrm{p}} T_{\mathrm{p}}^{-3 / 2}$. The collisional age is defined by the ratio of expansion and collisional time scales. Although the proton-proton collisional age is not representative for the collisional properties of the entire solar-wind plasma, we consider the proton-proton collisional age as a representative marker for the solar-wind stream type. (3) Only streams that remained within the respective same categorization regimes of the average $\mathrm{O}$ and $\mathrm{C}$ charge states and collisional age for at least half a day were considered. Fluctuations in $a_{\mathrm{col}}, n_{\mathrm{O}^{7+}} / n_{\mathrm{O}^{6+}}, n_{\mathrm{C}^{6+}} / n_{\mathrm{C}^{5+}}$, or the average Fe charge-state on smaller time scales than the four hours were permitted as long as the average value (averaged over four hours) remained in the respective range.

As a comparison baseline we also require pure slow solar wind. In this context, pure slow solar wind is characterized by high $\mathrm{O}$ charge states $n_{\mathrm{O}^{7+}} / n_{\mathrm{O}^{6+}}>0.1$, high $\mathrm{C}$ charge states
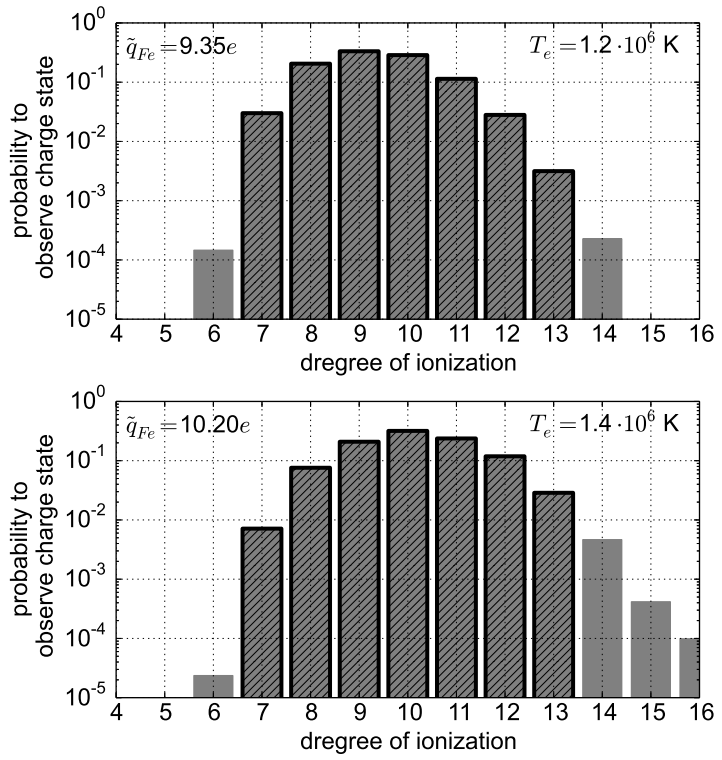

Fig. 1. Top panel: Fe charge-state distribution at $T=1.2 \times 10^{6} \mathrm{~K}$ taken from the CHIANTI database. Bottom panel: Fe charge-state distribution at $T=1.4 \times 10^{6} \mathrm{~K}$ from the CHIANTI. The black borders indicate the $\mathrm{Fe}$ charge states considered in this work. In both panels, the mean $\mathrm{Fe}$ charge-state $\tilde{q}_{\mathrm{Fe}}$ is given as inset on the left.

$n_{\mathrm{C}^{6+}} / n_{\mathrm{C}^{5+}}>1$, and high collisional age $a_{\mathrm{col}}>0.4$. This characterization of slow solar wind is not directly complementary to the criteria for identifying coronal-hole wind as described above in order to reduce the contamination of each wind type by transition regions that exhibit a mixture of properties of slow and coronal-hole wind.

In this study, we are interested in the Fe charge-state distribution in coronal-hole wind. The densities of the Fe charge states are provided by ACE/SWICS. In particular, since the most abundant Fe charge states are well isolated from all other ions in the SWICS $m-m / q$ diagram, we focus on these, namely $\mathrm{Fe}^{7+}, \mathrm{Fe}^{8+}$, $\mathrm{Fe}^{9+}, \mathrm{Fe}^{10+}, \mathrm{Fe}^{11+}, \mathrm{Fe}^{12+}$, and $\mathrm{Fe}^{13+}$. In the following the average Fe charge-state $\tilde{q}_{\mathrm{Fe}}$ is defined as $\tilde{q}_{\mathrm{Fe}}=\sum_{c=7}^{13} c n_{\mathrm{Fe}^{c+}} / \sum_{c=7}^{13} n_{\mathrm{Fe}^{c+}}$.

Figure 1 shows the Fe charge-state distribution for two electron temperatures $T=1.2 \times 10^{6} \mathrm{~K}$ (top) and $T=1.4 \times$ $10^{6} \mathrm{~K}$ (bottom) as provided by the atomic database CHIANTI (Dere et al. 1997; Landi et al. 2013). The temperatures are chosen from the typical range of observed electron temperatures in the corona (see e.g. Ko et al. 1997; Wilhelm 2012). The charge states considered here $\left(\mathrm{Fe}^{7+}, \mathrm{Fe}^{8+}, \mathrm{Fe}^{9+}, \mathrm{Fe}^{10+}, \mathrm{Fe}^{11+}, \mathrm{Fe}^{12+}\right.$, and $\left.\mathrm{Fe}^{13+}\right)$ are highlighted by hatched bars and black borders around the respective bars and are at the relevant temperatures the most prominent charge states.

Based on the CHIANTI data displayed in Fig. 1 and under the assumptions that the freeze-in temperature $T_{f, \mathrm{Fe}}$ for all Fe ions is the same and that $T_{f, \mathrm{Fe}} \sim 1.2 \times 10^{6} \mathrm{~K}$, Fig. 1 also illustrates that $q_{\mathrm{Fe}}=9$ would be the most likely charge state, with a mean charge state of 9.35 . For $T=1.4 \times 10^{6} \mathrm{~K}$ a mean charge state of 10.2 would be expected. However, since the assumption that all Fe ions freeze-in at the same temperature is not accurate, this provides only a rough guideline. Furthermore CHIANTI makes the assumption of a Maxwellian distribution for the electron velocity distribution function which is known not to be the appropriate choice for the solar corona and the solar wind (see for example, Marsch 2006). Instead our notion of high or low average Fe charge state is based on a comparison 
of Fe charge states in coronal-hole wind to those observed in selected samples of slow solar wind.

For 2004 the median of the average Fe charge-state of pure slow solar wind was $\tilde{q}_{\mathrm{Fe} \text {,slow }}=9.87$. The $1 \sigma$-level is bounded below by $\tilde{q}_{\mathrm{Fe} \text {,slow }}-\sigma=9.54$. These values are used to define our notion of high and low Fe charge states. We consider the average Fe charge state $\tilde{q}_{\mathrm{Fe}, \mathrm{CH}}$ of a coronal-hole-wind stream as low and the stream as Fe-cool if $\tilde{q}_{\mathrm{Fe}, \mathrm{CH}}<\tilde{q}_{\mathrm{Fe} \text {,slow }}-\sigma$. Analogously, we consider a coronal-hole-wind stream to be Fe-hot if its average Fe charge-state is within one $\sigma$ of the average Fe charge state of pure slow solar wind for that year or higher: $\tilde{q}_{\mathrm{Fe}, \mathrm{CH}}>\tilde{q}_{\mathrm{Fe}, \text { slow }}-\sigma$. Thus for 2004, the threshold value is $\tilde{q}_{\mathrm{Fe} \text {,slow }}-\sigma=9.54$ and for $2006 \tilde{q}_{\mathrm{Fe} \text {,slow }}-\sigma=9.71$.

To ensure that the selected coronal-hole-wind streams are not contaminated with interplanetary coronal mass ejections (ICMEs), we cross-referenced the Jian et al. (2006, 2011) and Richardson \& Cane (2010) ICME lists and the Large Angle and Spectrometric Coronagraph (LASCO) CME list and excluded all time periods with ICMEs from our times of interest. For four days after each halo CME in the LASCO list (Yashiro et al. 2004; Gopalswamy et al. 2009) that does not have a counterpart in the ICME lists, we verified that no ICME signatures were contained in the coronal-hole-wind stream candidates. Also, to reduce the effect of inter-stream regions, time periods with enhanced proton density and magnetic field strength which indicate stream interaction-regions (Jian et al. 2006, 2011) were excluded as well. With this method, 4660 12-min observations were selected as coronal-hole wind in 2004, accumulating to 38.8 days of combined coronal-hole wind, and 8346 observations corresponding to 69.5 days of combined coronal-hole wind in 2006. For the sake of an unbiased representation, we randomly chose three transitions from two different years between Fe-cool and Fe-hot coronal-hole wind from the available data set to be discussed in detail in the following section: day of year (DoY) 2-14 in 2004 and DoY 158-162 and DoY 212-215 in 2006. Six additional transitions in 2006 are mentioned briefly.

\section{Fe charge states of individual coronal-hole-wind streams}

We now focus on the Fe charge-state distribution of individual coronal-hole-wind streams and relate the average Fe charge state of these to the average Fe charge-state of all slow solar wind streams of the same year.

Figure 2 summarizes the solar wind properties for 12 days in 2004. From top to bottom, the panels in Fig. 2 show the collisional age $a_{\mathrm{col}}$, the ratios $n_{\mathrm{O}^{7+}} / n_{\mathrm{O}^{6+}}$ and $n_{\mathrm{C}^{6+}} / n_{\mathrm{C}^{5+}}$, the corresponding freeze-in temperatures $T_{f, \mathrm{O}^{7+} / \mathrm{O}^{6+}}$ and $T_{f, \mathrm{C}^{6+} / \mathrm{C}^{5+}}$, the magnetic field strength $B$ and angles $B_{\phi}, B_{\theta}$, and in the bottom panel the proton speed $v_{\mathrm{p}}$ (left $y$-axis) and proton density $n_{\mathrm{p}}$ (right $y$-axis). A coronal-hole stream (DoY 3.5-6.5) is followed by an interface region with a higher-speed stream and an ICME beginning on DoY 9. The ICME period is marked with gray hatching. From DoY 3.5 to DoY 6.5, $n_{\mathrm{O}^{7+}} / n_{\mathrm{O}^{6+}}$ and $n_{\mathrm{C}^{6+}} / n_{\mathrm{C}^{5+}}$ are low and, in particular, are below their respective thresholds for coronal-hole wind. The collisional age $a_{\text {col }}$ is below its threshold value of 0.1 for coronal-hole wind as well. Thus, according to the criteria described in Sect. 2, this suffices to categorize this stream as fast, that is, as coronal-hole wind. This is supported by the additional data products shown in Fig. 2. Although there is some variability in the solar-wind proton speed $v_{\mathrm{p}}$, the minimal value is still unlikely to be produced by slow solar wind. The freeze-in temperatures are derived under the assumption of an equilibrium state that allows us to relate the observed abundance ratio of two adjacent ions to the respective ionization and recombination rates: $n_{i} / n_{i+1}=R_{i+1}\left(T_{f}\right) / C_{i}\left(T_{f}\right)$. Since they depend on the density ratios $n_{\mathrm{O}^{7+}} / n_{\mathrm{O}^{6+}}$ and $n_{\mathrm{C}^{6+}} / n_{\mathrm{C}^{5+}}$ it is not surprising that the freeze-in temperatures are low during the coronal-hole-wind stream as well.

Although still below the threshold for coronal-hole wind defined above, the $n_{\mathrm{O}^{7+}} / n_{\mathrm{O}^{6+}}$ ratio and the collisional age are higher from DoY 3.5 to DoY 4.25 than in the following period. To prevent any interference by other processes, we focus on the part of the coronal-hole-wind stream from DoY 4.5-6.5 where both density ratios are safely below their respective categorization thresholds. Based on these considerations, the time period from DoY 4.5-6.5 (which is indicated with the gray shaded area in Fig. 2) contains only typical, quiet coronal-hole wind.

The ICME period exhibits a much larger variability in all data products; in particular, the $n_{\mathrm{O}^{7+}} / n_{\mathrm{O}^{6+}}$ and the collisional age are much higher. The magnetic-field angles show a smooth rotation indicating a magnetic cloud as part of the ICME. Thus, this ICME can easily be distinguished from the coronal-hole-wind stream.

Figure 3 focuses on the highlighted part of the coronal-holewind stream (DoY 4.5-6.5) from Fig. 2. For this time period of interest, Fig. 3 examines how the individual $\mathrm{Fe}$ charge states behave during DoY 4.5-5.6 and DoY 5.6-6.5. The motivation for this partition is detailed in Fig. 4. The upper five panels of Fig. 3 show the density ratios of $\mathrm{C}, \mathrm{O}$, and $\mathrm{Fe}$ ion pairs within these two parts of the coronal-hole-wind stream of interest, and the bottom panel provides examples of the charge-state distributions of $\mathrm{C}$, $\mathrm{O}$, and $\mathrm{Fe}$ for two selected observations. The vertical lines in the upper panels indicate the corresponding times used in the bottom panel. The horizontal lines in each panel give the mean density ratio in the left and right interval, respectively. All Fe ion density ratios shown here are higher in the first part of the stream than in the second part. Comparing the two charge-state distribution examples at the bottom, in the example on the left, not only is the maximum of the $\mathrm{Fe}$ charge-state distribution shifted from $\mathrm{Fe}^{9+}$ to $\mathrm{Fe}^{10+}$ as compared to the second example, but the complete distribution is shifted to higher charge states. Thus, a change in the mean charge-state cannot be explained by a single enhanced or depleted charge-state. Instead all considered charge states are affected.

As a continuous representation of the charge-state distributions in the bottom panel of Fig. 3, Fig. 4 shows a time series of the Fe charge-state distribution (considering only $\mathrm{Fe}^{7+}$ to $\mathrm{Fe}^{13+}$ ) in the first panel. The three bottom panels provide the solar-wind proton speed $v_{\mathrm{p}}$, proton density $n_{\mathrm{p}}$, and the ratio $n_{\mathrm{O}^{7+}} / n_{\mathrm{O}^{6+}}$ as reference. During DoY 4.5-5.6 the charge-state distribution of $\mathrm{Fe}$ is shifted to higher charge states more similar to those observed in slow solar wind. A transition between Fe-hot and Fe-cool coronal-hole wind (marked with a vertical black line) occurs at DoY 5.6. After the transition, at 5.6-6.5, lower Fe charge states are observed. Both the characterization as Fe-cool or Fe-hot wind and the resulting transition point are defined on four-hour resolution data. The same transition divides the left and right parts of the five top panels in Fig. 3. It is interesting to note that the Fe-hot part of the stream coincides with an average solar wind speed below $600 \mathrm{~km} \mathrm{~s}^{-1}$, whereas the Fe-cool interval shows an average solar wind speed higher than $600 \mathrm{~km} \mathrm{~s}^{-1}$. This hints at a potential stream boundary between two high-speed streams that coincides with the transition between Fe-hot and Fe-cool coronal-hole wind as a possible explanation for the observed transition. 

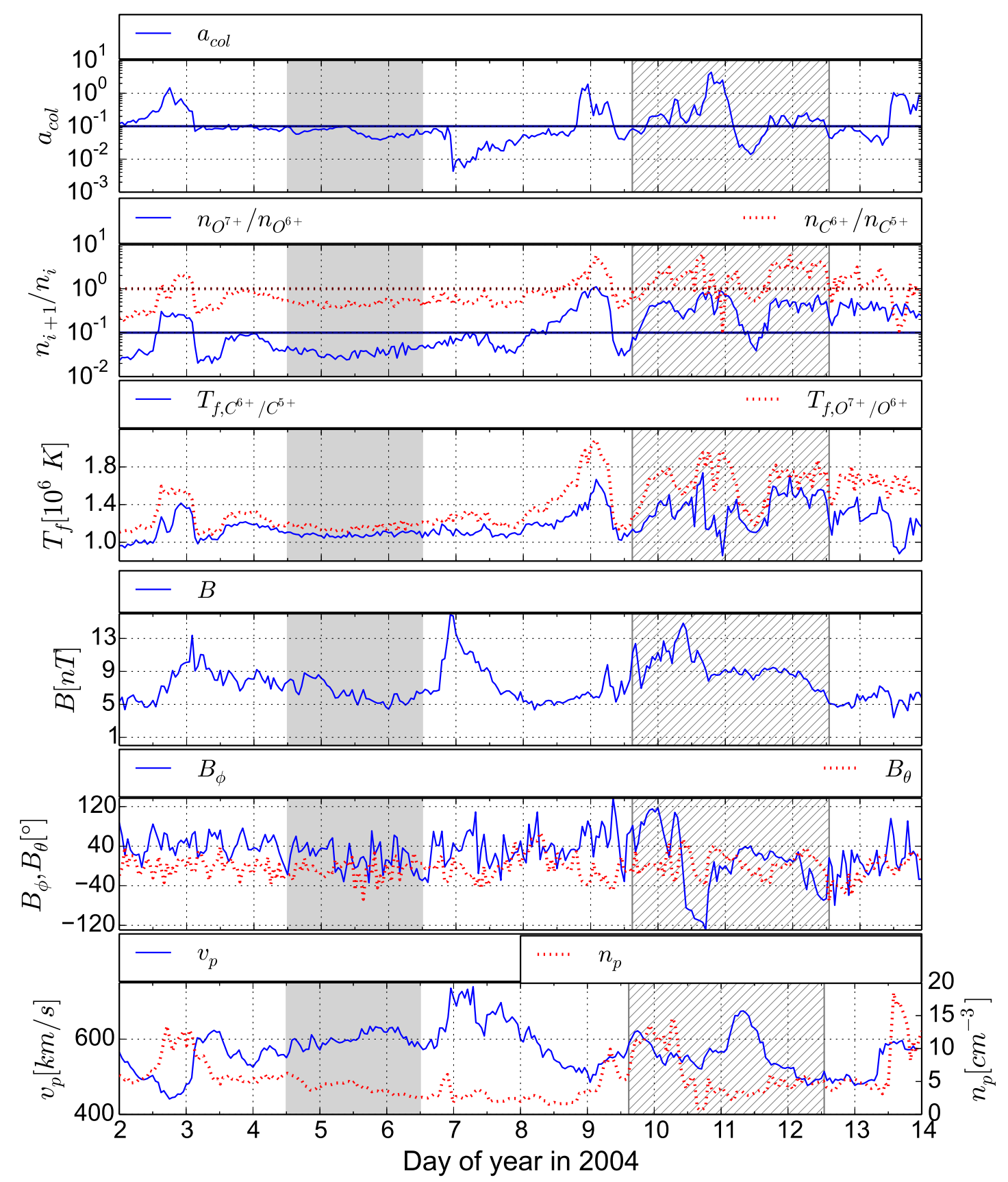

Fig. 2. Solar wind properties for 12 days in 2004. The collisional age $a_{\mathrm{col}}$ is displayed in the first panel. The second panel shows the density ratios $n_{\mathrm{O}^{7+}} / n_{\mathrm{O}^{6+}}$ and $n_{\mathrm{C}^{6+}} / n_{\mathrm{C}^{5+}}$. The horizontal lines in the first two panels indicate the respective selection thresholds for $a_{\mathrm{col}}=0.1, n_{\mathrm{C}^{6+}} / n_{\mathrm{C}^{5+}}=1$, and $n_{\mathrm{O}^{7+}} / n_{\mathrm{O}^{6+}}=0.1$. The third panel contains the freeze-in temperatures $T_{f}$ corresponding to the ion density ratios $n_{\mathrm{O}^{7+}} / n_{\mathrm{O}^{6+}}$ and $n_{\mathrm{C}^{6+}} / n_{\mathrm{C}^{5+}}$. The fourth panel gives the magnitude of the magnetic field $B$ and the azimuthal $\left(B_{\phi}\right)$ and polar $\left(B_{\theta}\right)$ angles of the magnetic field are shown in the fifth panel. The sixth panel shows the solar-wind proton speed (left $y$-axis) and proton density (right $y$-axis) as measured by SWEPAM. All data products are displayed with $1 \mathrm{~h}$ time resolution. The gray shaded area marks a pure coronal-hole-wind stream, while the hatched area highlights an ICME.

Figure 5 shows observations for a solar wind stream in 2006 which exhibits a transition from $\mathrm{Fe}-\mathrm{cool} \mathrm{CH}$ wind to $\mathrm{Fe}-\mathrm{hot}$ $\mathrm{CH}$ wind. In the top panel of Fig. 5, a time series of the chargestate distribution is shown (in the same way as in Fig. 4). An increase of the average Fe charge state is visible at DoY 160.4 thus indicating a transition from Fe-cool coronal-hole wind to Fe-hot coronal-hole wind. The panels below show the solar-wind proton speed, proton density, and the $n_{\mathrm{O}^{7+}} / n_{\mathrm{O}^{6+}}$ ratio measured at ACE. To allow a direct comparison with the model polarity in Fig. 6, the bottom panel gives additionally the magnetic-field polarity observed with ACE/MAG (Smith et al. 1998) for the same time period. In order to determine the in-situ magnetic-field polarity, we first derive the nominal magnetic-field direction $B_{\phi}^{\text {nom }}$, that is, the angle between the field line and the radial direction: $B_{\phi}^{\text {nom }}=\arccos \left(\sqrt{\frac{1}{1+\left(\omega r \sin (\theta) / v_{\mathrm{p}}\right)^{2}}}\right)$. Here, $\omega$ is the solar angular velocity, $r$ is the Sun-spacecraft distance, $\theta$ is the heliographic latitude and $v_{\mathrm{p}}$ is the in-situ solar-wind proton speed. Next, we subtract the nominal magnetic field angle $B_{\phi}^{\text {nom }}$ from the in-situ measured angle $B_{\phi}$. If the absolute difference is greater than $90 \mathrm{deg}$, the magnetic-field polarity is inwardly $(\otimes$, red) directed; otherwise it is outwardly $(\odot$, green) directed. A switch from outwardly 

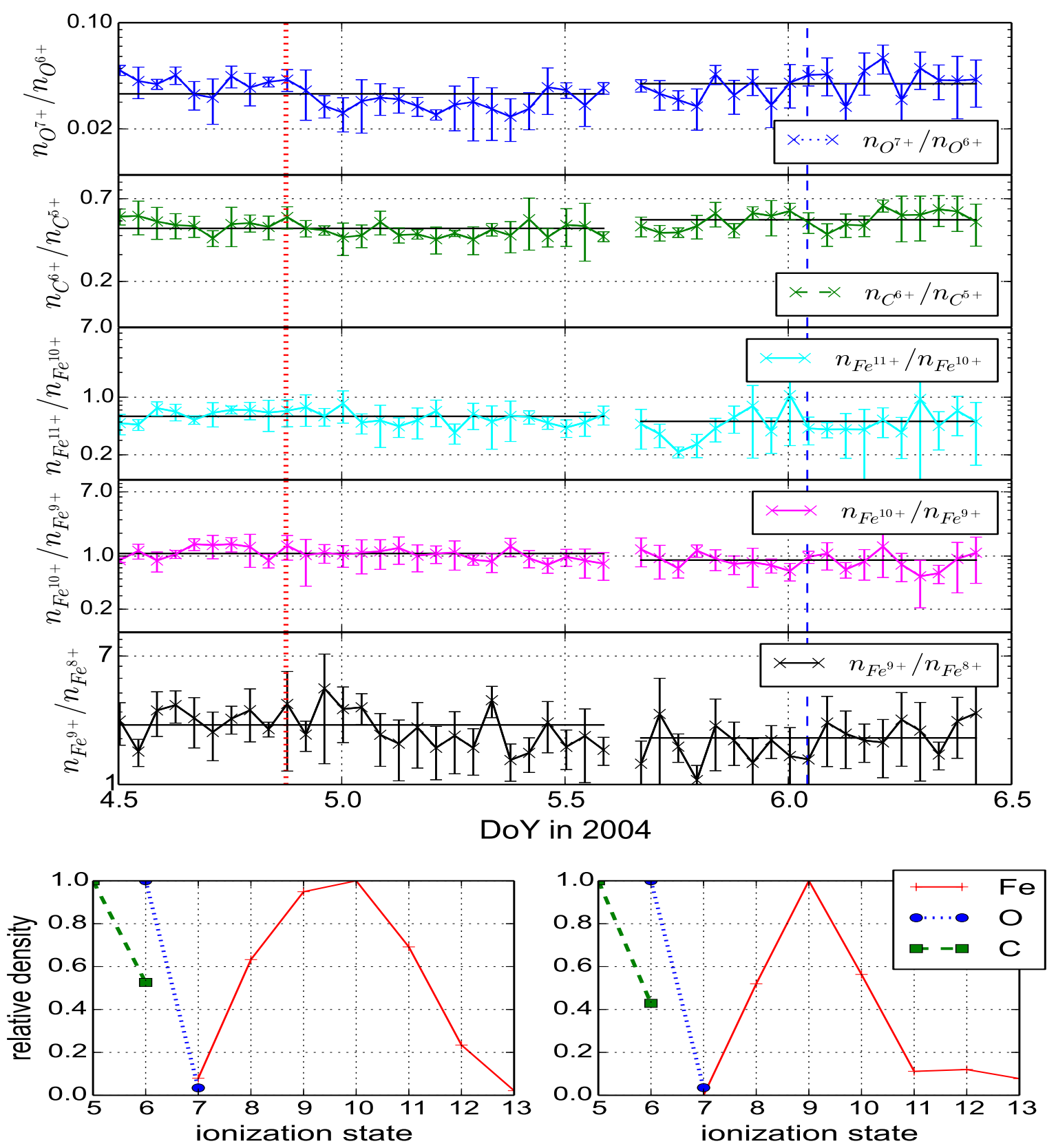

Fig. 3. Ion density ratios for the highlighted part of the coronal-hole-wind stream from Fig. 2. The five top panels show the density ratios of ion pairs, namely $n_{\mathrm{O}^{7+}} / n_{\mathrm{O}^{6+}}, n_{\mathrm{C}^{6+}} / n_{\mathrm{C}^{5+}}, n_{\mathrm{Fe}^{11+}} / n_{\mathrm{Fe}^{10+}}, n_{\mathrm{Fe}^{10+}} / n_{\mathrm{Fe}^{9+}}, n_{\mathrm{Fe}^{9+}} / n_{\mathrm{Fe}^{8+}}$. Each curve is divided into two parts. This partition is based on the mean Fe charge-state as illustrated in Fig. 4. The horizontal lines in each panel give the mean density ratio in the left and right interval, respectively. The vertical lines in the five top panels indicate the points in times for which examples of charge-state distributions normalized to the maximum density for $\mathrm{C}, \mathrm{O}$, and $\mathrm{Fe}$ are shown in the bottom panel. The dotted line corresponds to the left bottom panel and the dashed line to the right panel. (Although $\mathrm{C}^{4+}$ is more abundant than $\mathrm{C}^{6+}$ it is omitted here because it tends to be contaminated with adjacent O ions in ACE/SWICS.) Each data point represents a one-hour average and the error bars reflect the error from the counting statistics.

pointing (i.e. $\odot$, green) polarity to inwardly pointing polarity (i.e. $\otimes$, red) occurs at DoY 157.3. From DoY 157.3 onwards, the polarity remains inwardly pointing $(\otimes$, red) with some exceptions. We verified that the exceptions are caused by kinks in the magnetic field which can be seen by a reversal of the ionproton differential streaming (Berger et al. 2011). In particular, the polarity does not change on DoY 160 which includes the transition from $\mathrm{Fe}$-cool coronal-hole wind to Fe-hot coronal-hole wind.

With a combination of ballistic back-mapping and a potential field source surface (PFSS, Schatten et al. 1969; Altschuler \& Newkirk 1969) model, the photospheric source region of the ACE observations from DoY 158-163 can be estimated. Based on the in-situ solar wind speed, the solar rotation, and the heliographic coordinates of ACE, the position of ACE in heliographic coordinates is mapped back to the source surface. Here, a simple PFSS model takes over and allows us to track the field lines down to the photosphere. A uniform grid with $1^{\circ}$ resolution is assumed at the source surface. Peleikis et al. (2015) gives a more detailed description of the method applied here. The accuracy of this approach is limited by the varying age of different parts of the underlying magnetograms that are composites of images from 27 days. In the following, this back-mapping is used to test whether the observed coronal-hole wind can be 


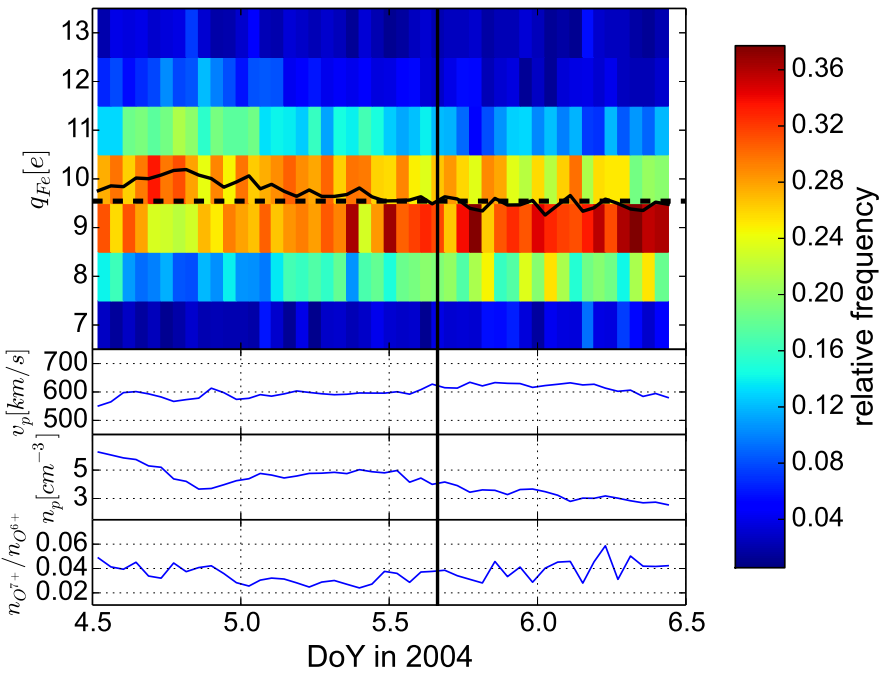

Fig. 4. Time series of Fe charge-state distributions (first panel) for the highlighted time period from Fig. 2, that is, DoY 4.5-6.5 in 2004, in one-hour time resolution. In black, the average charge-state (that is, $\left.\tilde{q}_{\mathrm{Fe}}=\sum_{c=7}^{13} c n_{\mathrm{Fe}^{c+}} / \sum_{c=7}^{13} n_{\mathrm{Fe}^{c+}}\right)$ is shown in units of the elementary electric charge $e$. Each charge-state distribution is normalized to the sum. Below, the solar wind proton speed (second panel) and proton density (third panel) are given as reference. In the bottom panel the ratio $n_{\mathrm{O}^{7+}} / n_{\mathrm{O}^{6+}}$ is shown as well. The threshold value between Fe-cool and Fe-hot wind is shown as a horizontal dashed line and the transition between Fe-hot and Fe-cool coronal-hole wind is marked with a vertical black line in all panels.

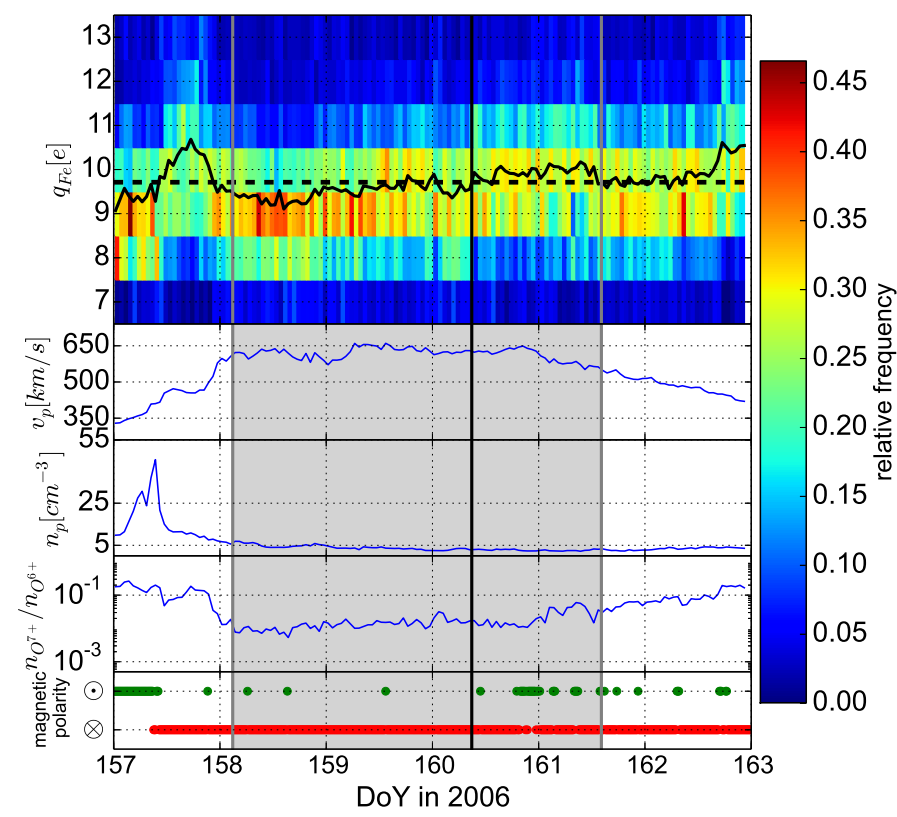

Fig. 5. Time series of the charge-state distributions of Fe for six days in 2006 with a one-hour time resolution and in the same format as in Fig. 4, with an additional panel for the magnetic-field polarity. Here, red indicates inwards pointing polarity $(\otimes)$ and green indicates outwardspointing polarity $(\odot)$.

associated with an open field line region in the photosphere and to investigate what kind of coronal structures are related to the transitions between Fe-cool and Fe-hot coronal-hole wind. Although only parts of the resulting heliographic maps are shown here, we examined the complete Carrington map in each case.

In Fig. 6, each cross in the lower panel represents a footpoint of a magnetic field line mapped back from the source

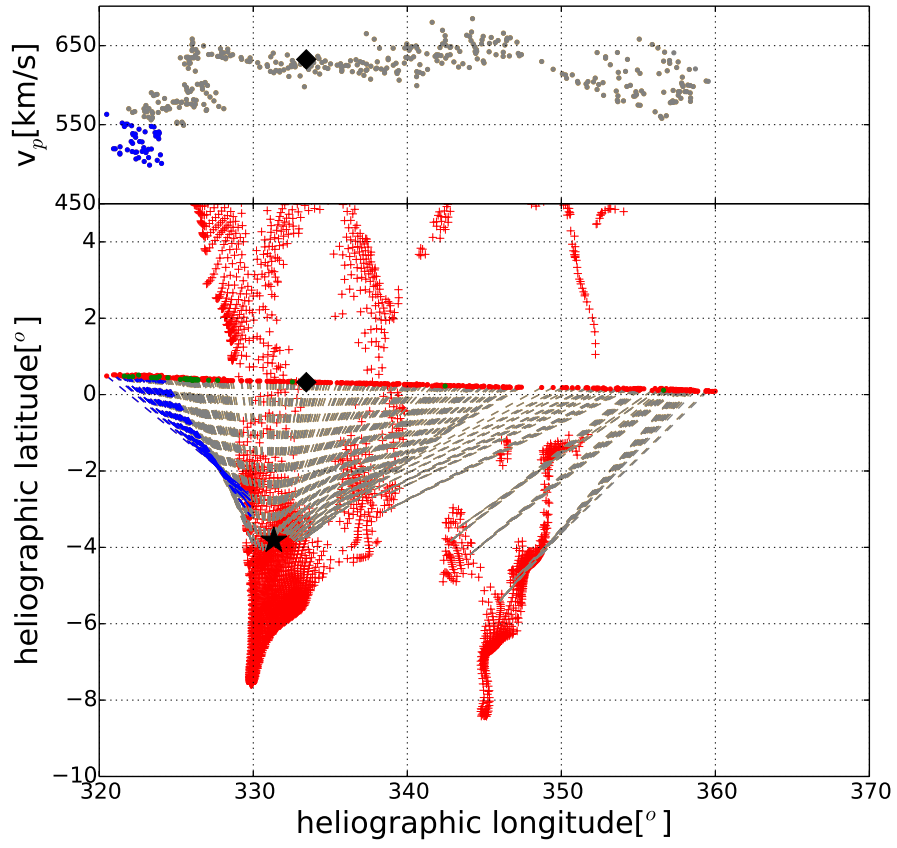

Fig. 6. Section of a heliographic map based on MDI magnetograms for Carrington rotation 2044. The first part of the highlighted part of the stream in Fig. 5 is omitted because it is part of the previous Carrington rotation. The top panel shows the solar-wind proton speed $\left(v_{\mathrm{p}}\right)$ plotted against the mapped back heliographic longitude on the source surface. Below, the dotted line shows the corresponding footpoints of ACE for DoY 158.4-162 on the source surface in heliographic coordinates. The color of each footpoint on the source surface corresponds to the magnetic-field polarity observed at ACE. The polarity is plotted in 12-min time resolution. These footpoints are then traced down to the photosphere with a PFSS model. The dashed lines connect the positions of the ACE footpoints on the source surface to the corresponding footpoints on the photosphere. Footpoints of open field lines in the photosphere are indicated with + symbols (red). The transition between the Fe-hot coronal-hole wind and the Fe-cool coronal-hole wind is marked with a black diamond on the source surface (and in the first panel) and with a black star on the photosphere.

surface down to the photosphere. Additionally for the corresponding Carrington rotation 2044, the footpoints of ACE are shown over this Carrington map derived from a PFSS model based on magnetrograms from the Michelson Doppler Imager (MDI, Scherrer et al. 1991) on the Solar and Heliospheric Observatory (SOHO). Figure 6 shows only the area to which the observations in the stream of interest in Fig. 5 are mapped back by the PFSS model. This area is an extension of the northern polar coronal hole to equatorial regions with - according to the PFSS model - red, inwardly pointing polarity. The dashed lines connecting the footpoints on the source surface and the photosphere corresponding to the coronal-hole-wind stream from DoY 158.12-161.59 are colored gray and the start of the subsequent declining phase of the stream (DoY 161.59-162) is colored blue. The border between the Fe-cool coronal-hole wind and the Fe-hot coronal-hole wind is indicated with a black diamond on the source surface and with a black star on the photosphere. The beginning of the period of interest belongs to the preceding Carrington rotation. Thus, for mapping the corresponding footpoints a different Carrington map would need to be considered. Since the transition itself occurs later in the stream and for the sake of clarity this first part of the stream is therefore omitted in Fig. 6. The remaining part of the coronal-hole-wind stream of interest, including the transition between the Fe-cool and the 


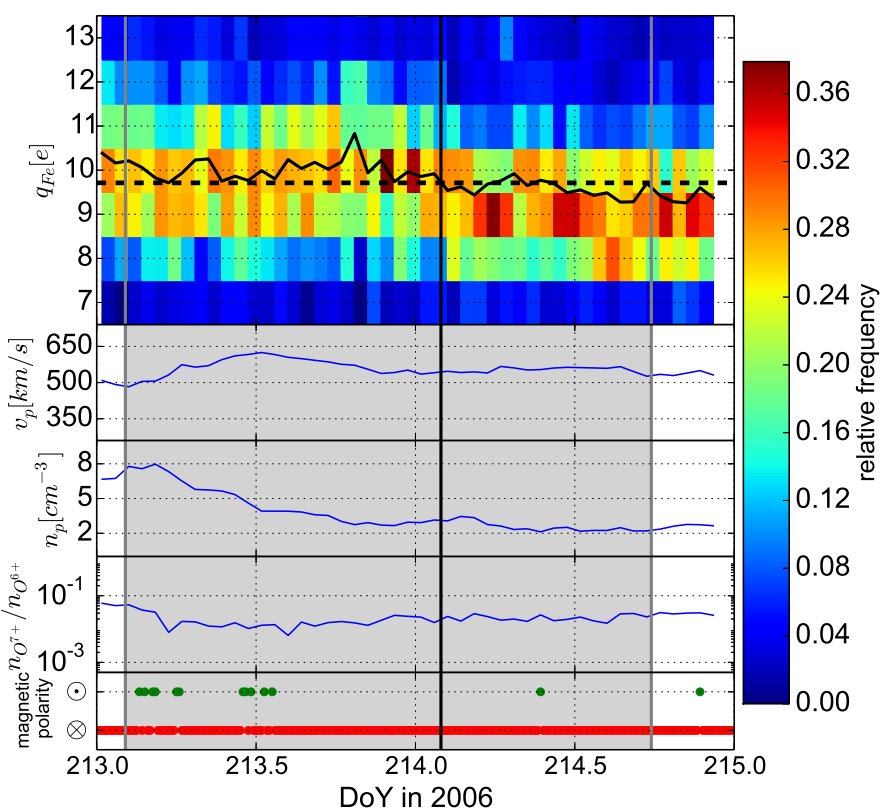

Fig. 7. Time series of the charge-state distributions of Fe for two days in 2006, with a one-hour time resolution (first panel) in the same format as in Fig. 5.

Fe-hot stream, and the following stream are mapped to a small scale region with open field lines in the equatorial region which is, as we verified on the complete Carrington map, connected to the northern polar coronal hole. For the whole time period and in particular, around the transition between the Fe-cool and Fe-hot stream, the in-situ observed polarities match (inwards pointing with the exception of the aforementioned kinks) the polarities predicted by the PFSS model. However, due to the limitations on the accuracy of the back-mapping caused by the requirement to derive the magnetic field lines from a complete Carrington rotation, the possibility that the transition occurs at an edge of this open field line region cannot be ruled out.

A second transition, DoY 213-215 in 2006, in this case from an Fe-hot to an Fe-cool stream, is shown as a charge-state distribution time series in Fig. 7. The Fe-hot part of this stream includes the beginning of the trailing edge of the high-speed stream as defined in Borovsky \& Denton (2016). The transition from $\mathrm{Fe}$-cool to Fe-hot wind, however, occurs $12 \mathrm{~h}$ earlier and is thus unlikely to be affected by the trailing edge. It is interesting to note that the Fe-hot part of the stream of interest includes faster solar wind than the Fe-cool part of the stream. However, the change in solar wind speed occurs $>5$ h earlier than the transition from Fe-hot to Fe-cool wind. Since the transition boundary is defined on four-hour averages, shifting the temporal bins cannot make the two changes coincide exactly but they might be closer, as it appears here. As shown in Fig. 8, which provides a part of the photospheric map for the relevant Carrington rotation 2046, the transition between the Fe-hot and Fe-cool coronal-hole-wind streams is situated within a larger region (compared to the case in Fig. 6) of open field lines. Inspection of the heliographic map for the complete Carrington rotation (not shown here) indicates that this region was not connected to a polar coronal-hole. In the following, we refer to such a region of open field lines which is not connected to a polar coronal-hole as isolated. Within the accuracy of the back-mapping approach, the transition lies within a region of open field lines. However, within this region, the field line density is not uniform; for example west of the transition (to the right of the star in Fig. 8) the field line density is decreasing at

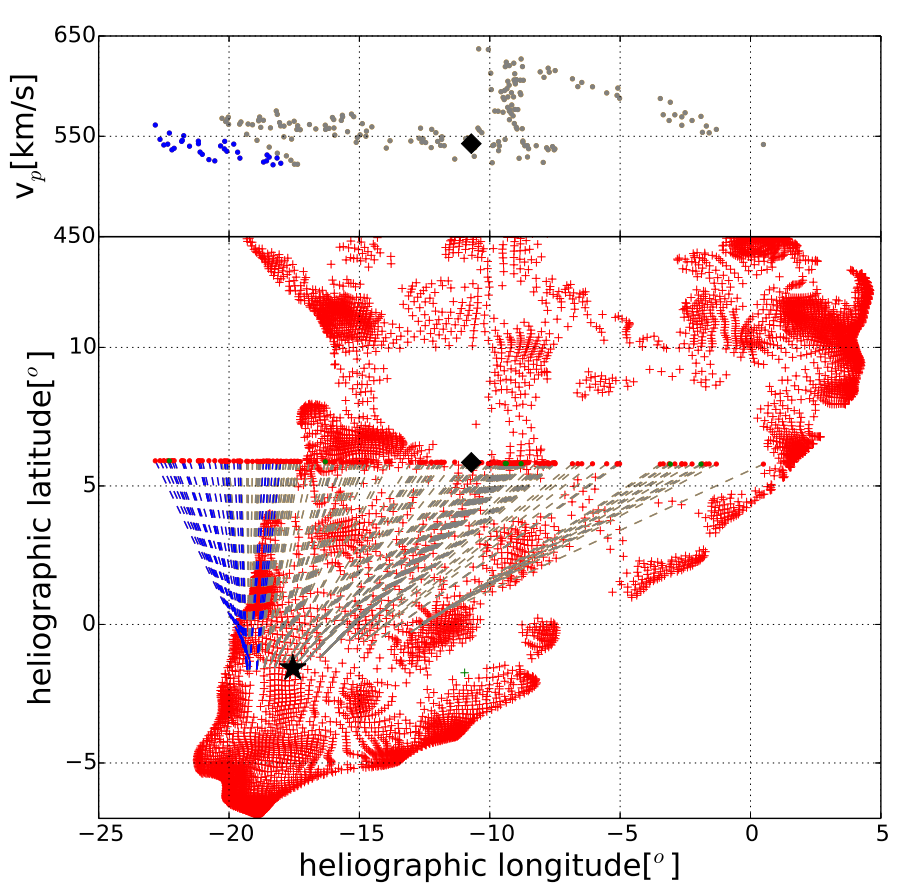

Fig. 8. A section of a heliographic map based on an MDI magnetogram for Carrington rotation 2046. The format is the same as in Fig. 6.

the photosphere. This fine-structure could be related to the transition. The in-situ magnetic polarity again matches the polarity predicted by the PFSS model with a few exceptions that can be explained as kinks in the magnetic field as well. For both transition examples in Figs. 6 and 8, both streams, in particular also the Fe-hot stream, originate in the equatorial region.

Table 1 summarizes the properties of eight transitions in 2006. The first column gives the start and end times of the part of the coronal-hole-wind stream containing the transition in DoY and the corresponding Carrington rotation is noted in the second column. An $*$ in the first column indicates the transitions shown in Figs. 5-8. The transition time is listed in the third column. To indicate the latitudinal and longitudinal position of the back-mapped foot points of each coronal-hole-wind stream, the highest and lowest back-mapped foot point is given in heliographic latitude in the fourth column, and the respective heliographic longitude in the fifth column. The last column indicates whether the stream is mapped to an isolated open field line region or whether the corresponding open field line region is connected to the northern polar coronal-hole (NPCH). All these coronal-hole-wind streams have inwardly pointing magnetic polarity as indicated by the symbol $\otimes$ in the last column. All eight transitions are mapped back to low latitudes and the longitudinal spread is at most $18^{\circ}$. As listed in Table 1, four out of eight transitions between $\mathrm{Fe}$-cool and Fe-hot coronal-hole wind in 2006 were mapped back to isolated coronal holes in the equatorial region. The remaining three transitions were observed in the equatorial region as well, but the respective coronal holes were connected to the northern polar coronal-hole. With respect to the long-term behavior, Zhao \& Landi (2014) observed that wind from polar coronal holes is predominantly $\mathrm{Fe}$-hot, while equatorial coronal-hole wind is predominantly Fe-cool. The case study shown here illustrates that, independently of their connection to a polar coronal-hole, Fe-hot coronal-hole-wind streams are hidden within the mainly Fe-cool equatorial coronal-hole wind.

A possible explanation for the change in the Fe charge states at transitions between Fe-cool and Fe-hot coronal-hole wind is 
A\&A 593, A70 (2016)

Table 1. Eight transitions between Fe-cool and Fe-hot coronal-hole-wind streams in 2006.

\begin{tabular}{rrrrrrrrrl}
\hline \hline $\begin{array}{r}\text { Stream of interest } \\
\text { [DoY in 2006] }\end{array}$ & CR & $\begin{array}{r}\text { Transition time } \\
{[\text { DoY in 2006] }}\end{array}$ & \multicolumn{2}{c}{$\begin{array}{c}\text { Latitude } \\
{\left[{ }^{\circ}\right]}\end{array}$} & & $\begin{array}{c}\text { Longitude } \\
{\left[{ }^{\circ} \text { ] }\right.}\end{array}$ & Isolated or connected \\
\hline $51.6-53.2$ & 2040 & 51.4 & -10.8 & to & -5.7 & 320.2 & to & 331.6 & $\otimes$ isolated \\
$104.9-107.0$ & 2042 & 105.7 & -5.0 & to & -4.0 & 317.5 & to & 320.9 & $\otimes$ connected to NPCH \\
$131.5-134.4$ & 2043 & 133.4 & -7.7 & to & -2.9 & 326.7 & to & 343.9 & $\otimes$ connected to NPCH \\
$* 158.1-161.6$ & 2044 & 160.4 & -5.4 & to & -2.4 & 329.8 & to & 348.5 & $\otimes$ connected to NPCH \\
$186.2-188.1$ & 2045 & 187.4 & -4.9 & to & -1.4 & 331.1 & to & 343.5 & $\otimes$ connected to NPCH \\
$* 213.1-214.5$ & 2046 & 214.1 & -1.7 & to & -2.9 & 341.0 & to & 351.7 & $\otimes$ isolated \\
$240.1-241.7$ & 2047 & 241.0 & -0.1 & to & 3.6 & 354.4 & to & 3.3 & $\otimes$ isolated \\
$294.0-296.0$ & 2049 & 295.0 & 5.2 & to & 5.7 & 4.9 & to & 5.7 & $\otimes$ isolated \\
\hline
\end{tabular}

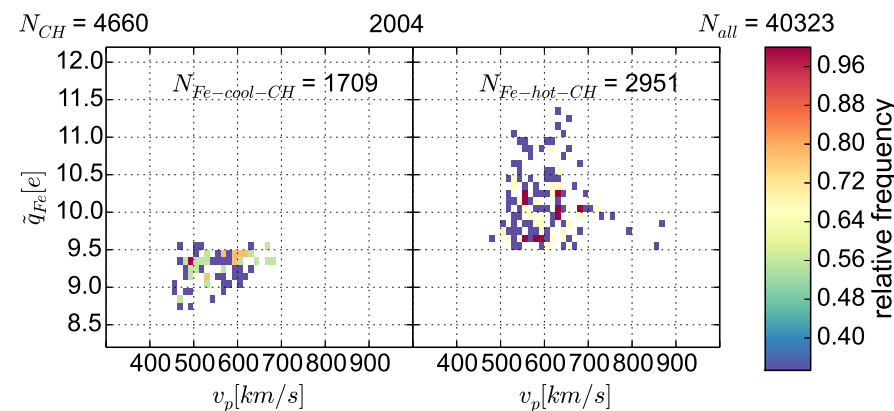

Fig. 9. Average charge-state $\left(\tilde{q}_{\mathrm{Fe}}\right)$ versus solar wind proton speed for $\mathrm{Fe}-\mathrm{cool}$ and Fe-hot coronal-hole type wind for 2004. The color gradient indicates the frequency of observing each charge-state-solar wind speed pair in 2004 and is normalized to the maximum in each panel. Each data point represents a four-hour average. The inset in each panel gives the number of data points in 12-min resolution that contributed to the averages in in this figure. The number $\left(N_{\text {all }}\right)$ of all 12-min resolution data points in 2004 is given on the top right and the number $\left(N_{\mathrm{CH}}\right)$ of all data points categorized as pure coronal-hole wind, in the top left.

that they coincide with stream interfaces between two distinct coronal-hole-wind streams. This is supported by the coinciding increase in solar wind speed and the decrease in proton density $n_{\mathrm{p}}$ in the 2004 example in Fig. 4. However, in Figs. 5 and 7, no clear change in the proton density $n_{\mathrm{p}}$ is visible at the transition. Furthermore, although the average solar wind speeds in the DoY 213-215 example in 2006 differ before and after the transition, the change in the solar wind speed does not coincide with the transition but occurs more than five hours earlier. Within the accuracy of the back-mapping approach, the back-mapped positions of the transitions in Figs. 6 and 8 might be consistent with stream interfaces at the transition times but this is not conclusive. Therefore, this explanation can neither be proved nor ruled out by the observations here.

To put the case studies presented above into some perspective, Figs. 9 and 10 illustrate how frequently Fe-cool and Fe-hot coronal-hole-wind streams were observed in 2004 and 2006, respectively. Figure 9 shows the frequency of the average charge-state versus the solar wind proton speed for all Fe-cool coronal-hole wind in 2004 (left panel) and all Fe-hot coronalhole wind in 2004 (right panel). The inset in each panel indicates the number of data points in 12-min resolution of Fe-cool $\left(N_{\mathrm{Fe}-\mathrm{cool}-\mathrm{CH}}\right)$ and Fe-hot coronal-hole wind $\left(N_{\mathrm{Fe}-\text { hot-CH }}\right)$ included in each panel. For reference, the number $N_{\text {all }}$ of all 12-min resolution data points in 2004 is given on the top right and the number $N_{\mathrm{CH}}$ of all data points categorized as pure coronal-hole wind in the top left. In the same way, Fig. 10 shows Fe-cool and

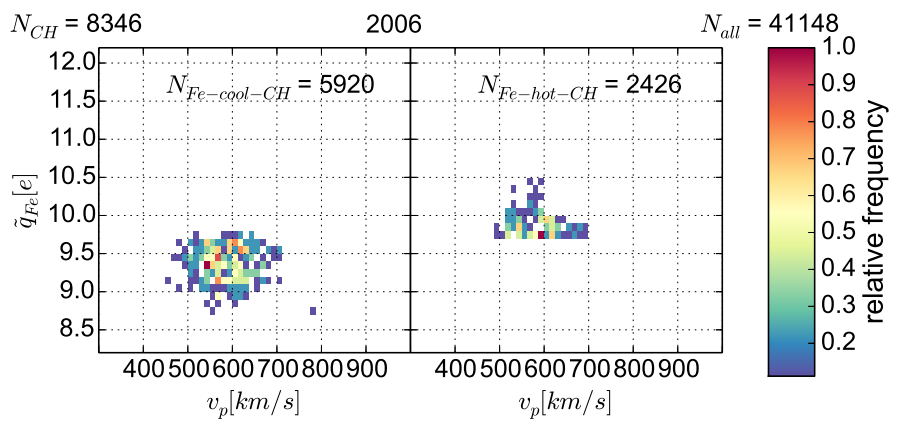

Fig. 10. Average charge-state $\left(\tilde{q}_{\mathrm{Fe}}\right)$ versus solar wind proton speed for $\mathrm{Fe}$-cool and $\mathrm{Fe}$-hot coronal-hole wind for 2006 in the same format as in Fig. 9.

Fe-hot coronal-hole wind for the year 2006. With respect to the solar wind speed, the distributions of both the Fe-cool and Fe-hot components of the coronal-hole wind overlap to a large extent in both years. In particular in 2004, the Fe-cool component contains slower solar wind than the Fe-hot component and the overlap is smaller than in 2006. The question under which conditions each type of coronal-hole wind is prevalent in other years is left for a later study. However, comparing the years 2004 (Fig. 9) and 2006 (Fig. 10) allows some observations. Firstly, while in 2004, 1709 individual observations can be identified as Fe-cool coronal-hole wind and 2951 as Fe-hot coronal-hole wind, the 2006 data contains $5920 \mathrm{Fe}$-cool coronal-hole wind observations and $2426 \mathrm{Fe}$-hot coronal-hole wind observations. This illustrates that in 2004 the Fe-hot wind is more frequent than the Fe-cool coronal-hole wind. In 2006, however, the opposite is the case: $\mathrm{Fe}$-cool coronal-hole wind is more frequent than Fe-hot coronalhole wind. Secondly, not only does the frequency of each wind type change but the Fe-hot coronal-hole wind is less variable in its average Fe charge state and the overall average Fe charge states are lower in 2006 than in 2004. (Although not shown here, this effect is not only visible for Fe but also for O.) Both observations hint at a solar-cycle dependence as observed for $\mathrm{O}$ and $\mathrm{C}$ in Kasper et al. (2012), Schwadron et al. (2011). Additionally, an overall drop of $\mathrm{O}$ and C charge states as observed in 2006, at the transition to the long solar minimum at the end of solar cycle 23 (e.g. Lepri et al. 2013; Richardson 2014), is probably superimposed on the Fe charge-state distribution as well. This aspect requires further investigation.

The 2004 data in Fig. 9 exhibits an interesting feature with respect to a possible solar-wind proton speed dependence of the average Fe charge state. The average Fe charge state of the $\mathrm{Fe}$-cool component (left panel) of the coronal-hole wind shows a possible dependence on the solar-wind speed. However, this is 
not visible in the Fe-hot component in the right panel which instead manifests a larger variability in the average Fe charge state for each solar-wind speed. For the 2006 example, this feature is not visible.

\section{Conclusions}

Complementing the observations of the long-term behavior of the Fe charge states in von Steiger et al. (2000), Galvin et al. (2009), Richardson (2014), Lepri et al. (2013), Kasper et al. (2012), Schwadron et al. (2011), Zhao \& Landi (2014), we present a case-study of individual solar-wind streams that can be clearly identified as coronal-hole wind, with either high or low Fe charge states compared to the charge states in slow solar wind. Streams with either property occur in the same year and we also observe direct transitions between them. This indicates that the steady coronal-hole wind is less uniform in terms of $\mathrm{Fe}$ charge states than with respect to $\mathrm{O}$ and $\mathrm{C}$ charge states. In particular, solar-wind streams with high $\mathrm{Fe}$ charge states are $\mathrm{Fe}-$ hot and $\mathrm{C} / \mathrm{O}-\mathrm{cool}$ at the same time. Under the assumption that the charge-state distribution is frozen-in in the corona, a higher Fe charge state implies a higher freeze-in temperature for the same cool $\mathrm{O}$ freeze-in temperature and thus, a qualitatively different temperature profile in the corona.

The back-mapping of transitions between $\mathrm{Fe}$-cool and $\mathrm{Fe}$-hot coronal-hole-wind streams finds the origin of both streams in equatorial regions and close to each other. Thus, streams with consistently high or low Fe charge states can originate in the same region. A more systematic investigation of the origin of all individual Fe-hot and Fe-cool coronal-hole-wind streams is beyond the scope of this case-study. In addition, we have seen that in $2004 \mathrm{Fe}$-hot coronal-hole wind is more frequent, while in $2006 \mathrm{Fe}$-cool coronal-hole wind is predominantly observed. A possible solar-cycle dependence of the frequency of Fe-hot and $\mathrm{Fe}$-cool coronal-hole wind - as it has been observed for $\mathrm{O}$ and $\mathrm{C}$ in Schwadron et al. (2011) and for Fe as well over solar cycle 23 in Lepri et al. (2013) - is one possibility to explain the changes in the frequency of Fe-hot and Fe-cool coronal-hole wind between 2004 and 2006. The details of a solar-cycle dependence, the evolution of the respective coronal structures over consecutive Carrington rotations, and the implications of these observations for the temperature profile in coronal holes require further investigation.
Acknowledgements. Part of this work was supported by the Deutsche Forschungsgemeinschaft (DFG) project number Wi-2139/10-1. We very gratefully acknowledge the diligent work of the anonymous referee who provided very detailed and helpful suggestions and comments.

\section{References}

Altschuler, M. D., \& Newkirk, G. 1969, Sol. Phys., 9, 131

Berger, L. 2008, Ph.D. Thesis, Kiel, Christian-Albrechts-Universität

Berger, L., Wimmer-Schweingruber, R., \& Gloeckler, G. 2011, Phys. Rev. Lett., 106,151103

Borovsky, J. E., \& Denton, M. H. 2016, J. Geophys. Res.: Space Phys., 121, 5055

Dere, K., Landi, E., Mason, H., Fossi, B. M., \& Young, P. 1997, A\&AS, 125, 149

Galvin, A., Popecki, M., Simunac, K., et al. 2009, Ann. Geophys, 27, 3909

Gloeckler, G., Cain, J., Ipavich, F., et al. 1998, in The Advanced Composition Explorer Mission (Springer), 497

Gopalswamy, N., Yashiro, S., Michalek, G., et al. 2009, Earth, Moon, and Planets, 104, 295

Jian, L., Russell, C., Luhmann, J., \& Skoug, R. 2006, Sol. Phys., 239, 393

Jian, L., Russell, C., \& Luhmann, J. 2011, Sol. Phys., 274, 321

Kasper, J., Lazarus, A., \& Gary, S. 2008, Phys. Rev. Lett., 101, 261103

Kasper, J., Stevens, M., Korreck, K., et al. 2012, ApJ, 745, 162

Ko, Y.-K., Fisk, L. A., Geiss, J., Gloeckler, G., \& Guhathakurta, M. 1997, Sol. Phys., 171, 345

Landi, E., Young, P., Dere, K., Del Zanna, G., \& Mason, H. 2013, ApJ, 763, 86

Lepri, S., Landi, E., \& Zurbuchen, T. 2013, ApJ, 768, 94

Marsch, E. 2006, Liv. Rev. Sol. Phys., 3, 1

McComas, D., Bame, S., Barker, P., et al. 1998, in The Advanced Composition Explorer Mission (Springer), 563

Peleikis, T., Kruse, M., Berger, L., Drews, C., \& Wimmer-Schweingruber, R. F. 2015, in Solar Wind 14: Proc. of the fourtheenth international Solar wind Conf., AIP Conf. Proc., 1720, 020003

Richardson, I. 2014, Sol. Phys., 289, 3843

Richardson, I., \& Cane, H. 2010, Sol. Phys., 264, 189

Schatten, K., Wilcox, J., \& Ness, N. 1969, Sol. Phys., 6, 442

Scherrer, P., Hoeksema, J., \& Bush, R. 1991, Adv. Space Res., 11, 113

Schwadron, N., Smith, C., Spence, H. E., et al. 2011, ApJ, 739, 9

Smith, C. W., L'Heureux, J., Ness, N. F., et al. 1998, in The Advanced Composition Explorer Mission (Springer), 86, 613

Tu, C., Zhou, C., Marsch, E., et al. 2005, Science, 308, 519

von Steiger, R. V., Schwadron, N., Fisk, L., et al. 2000, J. Geophys. Res.: Space Phys., 105, 27217

Wilhelm, K. 2012, Space Sci. Rev., 172, 57

Yashiro, S., Gopalswamy, N., Michalek, G., et al. 2004, J. Geophys. Res.: Space Phys., 109

Zhao, L., \& Fisk, L. 2010, in SOHO-23: Understanding a Peculiar Solar Minimum, ASP Conf. Ser., 428, 229

Zhao, L., \& Landi, E. 2014, ApJ, 781, 110

Zhao, L., Zurbuchen, T. H., \& Fisk, L. A. 2009, Geophys. Res. Lett., 36, L14104

Zurbuchen, T., Fisk, L., Gloeckler, G., \& von Steiger, R. 2002, Geophys. Res. Lett., 29, 66 